\title{
A REDUCED-STATE SISO ALGORITHM FOR MULTILEVEL MODULATION IN TURBO EQUALIZATION
}

\author{
M. Magarini, L. Reggiani, and A. Spalvieri \\ Dipartimento di Elettronica e Informazione, Politecnico di Milano, \\ Piazza Leonardo da Vinci, 32, I-20133 Milano (Italy), \\ \{magarini,reggiani,spalvier\}@elet.polimi.it
}

\begin{abstract}
Turbo equalization is an iterative equalization decoding scheme for detecting encoded data transmitted over channels that introduce intersymbol interference. In such a scheme complexity of the optimal soft-input soft-output (SISO) equalizer, which implements the BCJR a posteriori probability computation algorithm, is one of the major concerns. In this paper we extend the reduced state sequence detection (RSSD) algorithm to a BCJR-SISO equalizer matched to a non binary modulation. The resulting reduced-state SISO (RS-SISO) algorithms can take full advantage from the flexibility offered by RSSD in reducing the number of trellis states. However, extension of RSSD algorithm for obtaining the RS-SISO equalizers requires attention in the recombination procedure of the forward and backward metrics.
\end{abstract}

Keywords - Iterative detection, intersymbol interference, turbo equalization, reduced-state trellis SISO equalization.

\section{INTRODUCTION}

Coding and interleaving are used in radio communication systems as a means to ensure reliable data transmission. When transmission takes place on frequency selective channels, which introduce intersymbol interference (ISI), the classical architecture of the receiver consists of the cascade of detector, de-interleaver, and decoder. It is well known that the performance of this architecture could be significantly improved by iterating the soft detection-decoding process, leading to the so called turbo equalizer (TEQ) scheme [1]. In the receiver implementation the complexity of the optimal soft-input soft-output (SISO) equalizer, which implements the BCJR a posteriori probability (APP) computation algorithm [2], is one of the major concerns. For M-ary phase shift keying (PSK) modulations (e.g. 8-PSK in EDGE, enhanced data rate for GSM evolution) and severely distorted channels, a reduced-state SISO (RS-SISO) equalizer is required for a practical implementation [2].

The main contribution of this work is the extension of the full flexibility offered by reduced state sequence detection (RSSD, [3]) to the trellises used to compute the forward and backward probabilities. RSSD is one of the most popular techniques for reducing complexity of the maximum likelihood sequence detector: a local decision feedback is used to approximate the Viterbi algorithm using a smaller trellis. The application of RSSD to reduce complexity of the BCJR algorithm has already been addressed in [4]; however, reduction of the number of states is presented only by means of the delayed decision feedback sequence detection (DDFSD) that is the special case of RSSD when the same memory reduction is operated for all the bits that constitute the symbol.

Apart from the straightforward extension to nonbinary modulation of the RS-SISO algorithm in [4], this paper deals also with the application of RSSD to the RS-SISO algorithm proposed in [5]. In [5] modulation is binary and the DDFSD algorithm is realized in forward and backward recursions preceded by different filters. The idea of using two different filters, instead of one, was first proposed in [6] to improve performance of the RSSD algorithm in combined equalization and decoding. Likewise in $[5]$ it is shown that a performance improvement can be obtained for TEQ schemes based on RS-SISO equalizers where this form of double filtering is implemented.

The paper is organized as follows. Section II introduces the system model. Section III resumes RSSD algorithm and the RS-SISO presented in [4]. In section IV we discuss how RSSD can be extended to RS-SISO equalization with double filtering. Section V presents simulation results that provide a significant performance comparison of the two RS-SISO algorithms in a TEQ scheme.

\section{System Model}

The transmitter consists of a convolutional encoder that is fed by a sequence of i.i.d. information bits. The resulting coded bits are interleaved, and groups of $q$ bits are mapped onto the $2^{q}$ signal points. The model is constituted by a complex-valued baseband equivalent frequency selective channel that introduces ISI and additive white Gaussian noise (AWGN).

The discrete-time signal at the output of the sampled 
matched filter at time $k$ is

$$
r_{k}=\sum_{i=-\nu}^{\nu} x_{i} \tilde{c}_{k-i}+n_{k}
$$

where $\left\{\tilde{c}_{k}\right\}$ is the sequence of transmitted symbols, $\left\{n_{k}\right\}$ is a zero mean Gaussian noise sequence with autocorrelation function $\left\{\frac{N_{0}}{2} x_{k}\right\}\left(\frac{N_{0}}{2}\right.$ is the two sided power spectral density of the noise), and

$$
x_{k}=x(k T)=\int_{-\infty}^{\infty} g(t) g(t+k T) d t
$$

is the sampled autocorrelation of the impulse response of the channel $g(t)$ ( $T$ is the symbol time interval). We assume that ISI affects a limited number of symbols, i.e. $x_{k}=0$ for $|k|>\nu$, where $\nu$ defines the memory of the channel measured in time symbol intervals.

Starting from the sequence given in (1) the BCJR equalizer calculates the APP of each transmitted symbol. In the BCJR algorithm the APP of each symbol is computed by combining two probabilities that come from two different recursions: one operating on a trellis in the forward direction and the other on a trellis in the backward one [2]. Reliability of each symbol is used by a block that computes the corresponding APP of the bits; the resulting bit sequence is de-interleaved and passed to the BCJR-SISO decoder. In each system the SISO algorithms, including those with reduced number of states, are realized by means of the APP algorithm in the logarithmic domain and the logarithm of the sum is computed using the max* approximation [2].

When an RS-SISO equalizer is implemented, it is of crucial importance to define an appropriate filtering of the observed sequence in order to limit performance loss due to state reduction. The appropriate form of filtering depends on the specific implementation of the RS-SISO algorithm. In the next two sections we present two relevant choices for the RS-SISO algorithms and, for each one, the type of filtering applied to the discrete-time signal (1).

\section{Reduced State Single Trellis SiSO Algorithm}

In RSSD a reduction of the number of states is operated at level $i$ of the channel memory (for element $c_{k-i}$ ) by partitioning the signal constellation into $J_{i}$ subsets using the Ungerboeck set partitioning principle [7]. Let $a_{k-i}$ be the subset associated to the symbol $c_{k-i}$. A trellis state $s(k)$ is defined by the concatenation of the respective subsets. Let $s_{k}=\left(a_{k-\nu+1}, \ldots, a_{k}\right)$ be the generic state at time $k$ in the reduced trellis. Throughout the paper we assume that $J_{0} \geq \cdots \geq J_{\nu}$; in addition the number of states of the RSSD trellis is given by $\prod_{i=0}^{\nu-1} J_{i}$. We define a state reduction pattern by using a vector $\boldsymbol{\mu}$ whose $q$ entries are the duration of the memories associated to each bit (from the most significant (MSB, first entry) to the least one (LSB, last entry)).

It is well known that in order to obtain good performance with RSSD equalization it is essential to operate an appropriate filtering of the received sequence [8]. In [5] the benefits of using the MMSE-DFE (minimum mean square error - decision feedback equalizer) FF (feedforward filter), over the noise whitening FF, has been shown also in RS-SISO equalization algorithms. The discretetime sequence at the output of the MMSE-DFE FF is

$$
y_{k}=\sum_{i=0}^{\nu} d_{i} \tilde{c}_{k-i}+w_{k}
$$

where $\left\{d_{k}\right\}$ is the overall impulse response and $\left\{w_{k}\right\}$ is a zero mean white distortion sequence (residual ISI plus noise) having variance $N_{0} / 2$. The analytic expressions of the impulse response coefficients $\left\{d_{k}\right\}$ and of the MMSEDFE FF impulse response can be found in [5].

It is important to observe that when the RSSD algorithm is applied to trellis reductions, partitioning of the signal constellation into subsets turns out to be different for the forward and the backward states. In fact, due to time reversal in the backward process, the generic subset $a_{k-\nu+1+i}$, associated to symbol $c_{k-\nu+1+i}$ in the backward trellis, assumes the same role of subset $a_{k-i}$, associated to symbol $c_{k-i}$ in the forward trellis $(i=0, \cdots, \nu)$. For some state reduction patterns, this could lead to an ambiguity in the combination of the forward and backward probabilities unless it is adopted the approach suggested in [4] where the backward recursion is computed over the state history achieved by the forward one. In this case the two recursions are computed on trellises where the forward and the backward states are defined by the same concatenation of subsets. Note that, in this case, the backward algorithm cannot start until the forward one is concluded, excluding any possibility of parallel computation.

The transition metric that diverges at time $k-1$ from state $s_{k-1}=\left(a_{k-\nu}, \ldots, a_{k-1}\right)$ and merges at time $k$ in state $s_{k}=\left(a_{k-\nu+1}, \ldots, a_{k}\right)$ is given by

$$
\begin{gathered}
\ln \gamma\left(s_{k-1}, s_{k}\right)=-\frac{1}{N_{0}}\left|y_{k}-\sum_{i=0}^{\nu} d_{i} c_{k-i}\left(s_{k-1}\right)\right|^{2} \\
+\ln P\left(s_{k} \mid s_{k-1}\right)
\end{gathered}
$$

where $c_{k-i}\left(s_{k-1}\right)$ is the estimate of the $(k-i)$-th PSK symbol identified by the survivor path and by the state $s_{k-1}$ and $P\left(s_{k} \mid s_{k-1}\right)$ is the a-priori transition probability between states $s_{k-1}$ and $s_{k}$ (in the turbo equalizer the extrinsic information assumes the role of a-priori probability). Of course local decision feedback requires selection of a single survivor path per state: as in [5], we choose as a survivor the path that gives the greatest contribution to the state probability. 


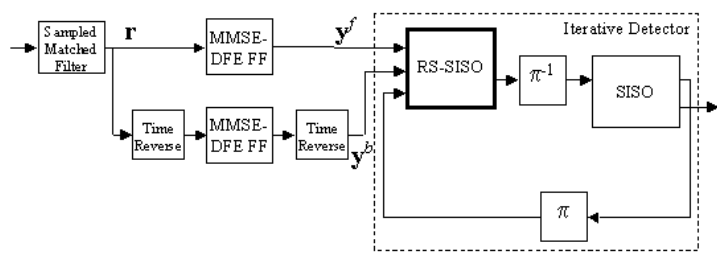

Fig. 1. TEQ scheme for the implementation of joint equalization and decoding.

\section{Reduced State Double Trellis SiSO ALGORITHM}

A different approach can be adopted to extend the RSSD for reducing complexity of the optimum BCJR equalizer. Following the design lines in [5], we apply RSSD technique to trellises where forward and backward states $s^{f}(k)$ and $s^{b}(k)$ are defined by the concatenation of the respective, possibly different, subsets; this does not limit backward recursion to the exploration of the path history accumulated in the forward stage. As stated in [5], a successful implementation of this approach needs a different filtering of the sequence (1) for the forward and the backward trellises; the clear goal is to make the impulse response $\left\{d_{k}\right\}$ minimum phase, maximizing the energy of the first tap weights. At the same time, in the backward process, signal energy in the last taps
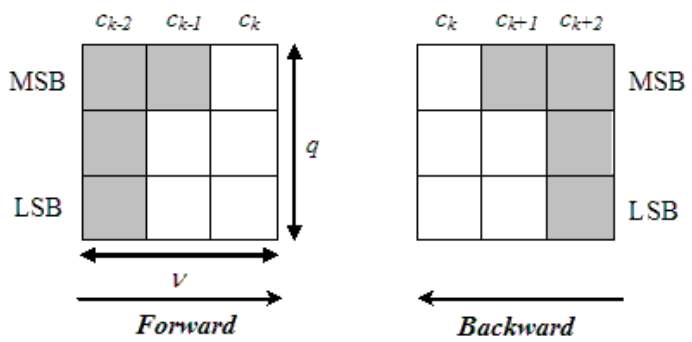

(a)

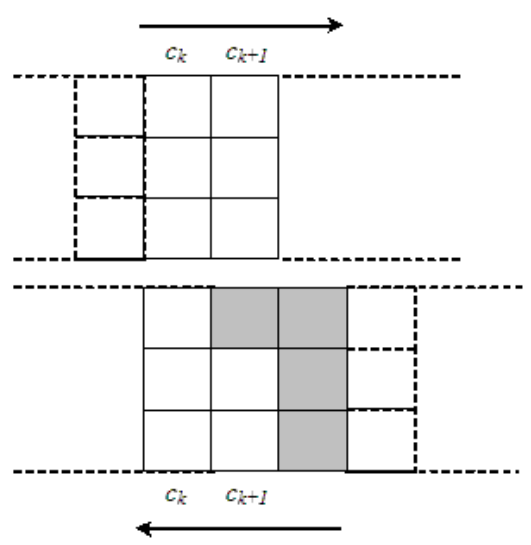

(b)

Fig. 2. In this example $\nu=3$ and $\boldsymbol{\mu}=\left[\begin{array}{lll}1 & 2 & 2\end{array}\right]$. should be maximized for improving the sub-optimal decision process. Hence the output of the matched filter is passed through a non-causal MMSE-DFE FF to obtain the sequence used in the forward recursion, while the time-reversed version of the mentioned filter is used for the backward recursion. So the impulse response used in the backward recursion turns out to be anti-causal and maximum phase. A pictorial description of the receiver scheme is reported in figure 1.

The signals at the input of the forward and backward trellises at time $k$ are respectively

$$
y_{k}^{f}=\sum_{i=0}^{\nu} d_{i} \tilde{c}_{k-i}+w_{k}^{f}, \quad y_{k}^{b}=\sum_{i=0}^{\nu} d_{i} \tilde{c}_{k+i}+w_{k}^{b},
$$

where $\left\{w_{k}^{f}\right\}$ and $\left\{w_{k}^{b}\right\}$ are zero mean white distortion sequences (residual ISI plus noise) having variance $N_{0} / 2$. Let $s_{k}^{f}=\left(a_{k-\nu+1}, \ldots, a_{k}\right)$ be the generic state at time $k$ in the reduced forward trellis, and let $s_{k}^{b}=\left(a_{k}, \ldots, a_{k+\nu-1}\right)$ be the generic state at time $k$ in the reduced backward trellis. The two transition metrics are given by

$$
\begin{gathered}
\ln \gamma\left(s_{k-1}^{f}, s_{k}^{f}\right)=-\frac{\left|y_{k}^{f}-\sum_{i=0}^{\nu} d_{i} c_{k-i}\left(s_{k-1}^{f}\right)\right|^{2}}{N_{0}} \\
+\ln P\left(s_{k}^{f} \mid s_{k-1}^{f}\right),
\end{gathered}
$$
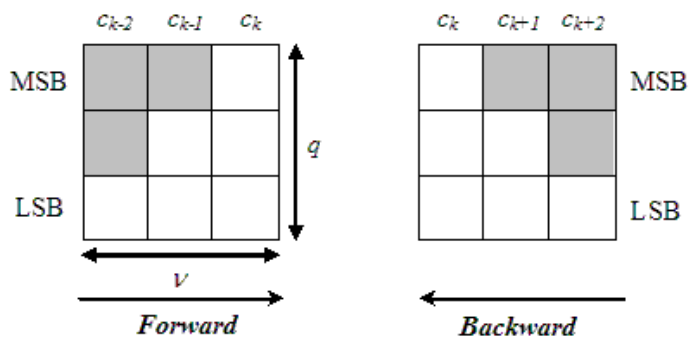

(a)

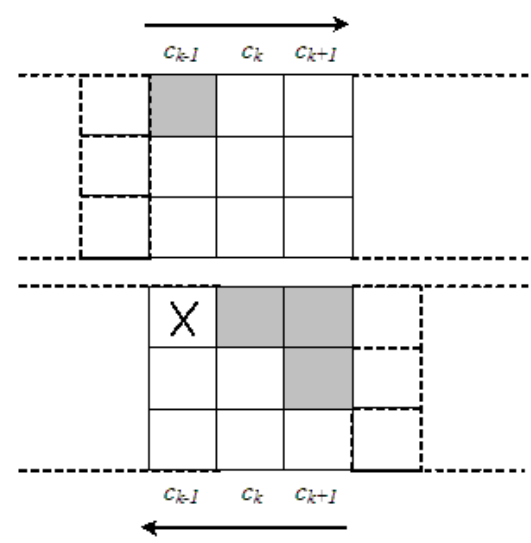

(b)

Fig. 3. In this example $\nu=3$ and $\boldsymbol{\mu}=\left[\begin{array}{lll}1 & 2 & 3\end{array}\right]$. 


$$
\begin{gathered}
\ln \gamma\left(s_{k+1}^{b}, s_{k}^{b}\right)=-\frac{\left|y_{k}^{b}-\sum_{i=0}^{\nu} d_{i} c_{k+i}\left(s_{k+1}^{b}\right)\right|^{2}}{N_{0}} \\
+\ln P\left(s_{k}^{b} \mid s_{k+1}^{b}\right)
\end{gathered}
$$

where the estimate of the $(k-i)$-th symbol $c_{k-i}\left(s_{k-1}^{f}\right)$ is identified by the survivor forward path and by the forward state $s_{k-1}^{f}$ and similarly $c_{k+i}\left(s_{k+1}^{b}\right)$ on the backward side; $P\left(s_{k}^{b} \mid s_{k+1}^{b}\right)$ and $P\left(s_{k}^{f} \mid s_{k-1}^{f}\right)$ are the a-priori probabilities associated to the current transitions. Note that the use of two FF's allows local decision feedback correction of the past symbols in the forward recursion and of the future ones in the backward one.

We emphasize here that extension of RSSD technique to this architecture is not straightforward because the recombination of the metrics between states of the two reduced trellises may not be defined unambiguously. This problem arises since RSSD is applied to trellises that proceed in opposite time directions using causal and anticausal impulse responses. We adopted a simple procedure that discriminates state reduction patterns $\mu$ that allow the dual metrics recombination without ambiguity: the resulting algorithm has to work on a trellis that has a fixed and pre-defined rule for recombining forward and backward metrics. To clarify this point two examples of reduced forward and backward states are represented in fig. 2-a and 3-a. The state $s_{k}^{f}$ and the generic transition, identified by the input symbol $c_{k+1}$, define not only the next forward state $s_{k+1}^{f}$ but also the backward state $s_{k-\mu+2}^{b}$ useful for the recombination. The memory of $s_{k}^{f}$ and $c_{k+1}$ are superimposed to the memory of a backward state until a depth equal to $\mu=\max \{\boldsymbol{\mu}\}$. The recombination is well defined when this superimposition brings to the exact identification of all the backward state bits (fig. 2-b); on the contrary, when the memory of $s_{k}^{f}$ and $c_{k+1}$ do not overlay all the memory bits of the backward state, recombination is not specifiable in a single way and the state reduction pattern is discarded (see the marked bit in fig. 3-b).

The reliability of the symbol transmitted at time $k$ is computed as [5]

$$
L\left(c_{k}\right)=\max _{c_{k}}\left\{\ln \alpha\left(s_{k-1}^{f}\right)+\ln \gamma\left(s_{k-1}^{f}, s_{k}^{f}\right)+\ln \beta\left(s_{k-\mu+1}^{b}\right)\right\}
$$

where $\alpha\left(s_{k-1}^{f}\right), \beta\left(s_{k-\mu+1}^{b}\right)$ are respectively the forward and the backward probabilities at time $k-1$ and $k-\mu+$ 1. Forward and backward probabilities are computed recursively, as usual [2], on the two filtered versions of signal (4).

\section{Simulation Results}

We are considering, as in [9], the possibility of using a TEQ for EDGE; the architecture of EDGE is the same as GSM, except for the use of 8-ary phase-shift keying (8-PSK) instead of binary Gaussian minimumshift keying (GMSK). The 8-PSK modulation is tested by means of a TEQ consisting of the RS-SISO equalizer, a random 4086 bit long interleaver and a convolutional code with rate $1 / 2$. Numerical results are presented for two channels: 1) a hard-to-equalize static channel ([5]) having discrete time autocorrelation func-

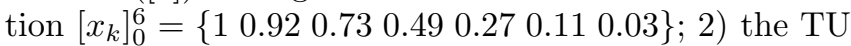
profile channel given in [10]. The channels are assumed to be perfectly known to the receiver; the former is a channel that remains static for all the block length while the latter changes at each block of 114 symbols modelling a fast fading hypothesis. The convolutional code, in octal representation, is $(133,171)$ for both channels. We have compared our state reduction strategy (denoted as DRT, double reduced trellis) with the solution without double filtering (SRT, single reduced trellis).

Fig. 4 reports word error rate (WER) achieved after three turbo iterations for the TU profile channel in a selection of reduction patterns; $E_{b}$ is the average received energy per information bit. We can observe that DRT architecture has a performance advantage that increases as the state reduction gets larger, achieving $0.5-0.75$ $\mathrm{dB}$ at $W E R=10^{-2}$ with 8-state equalizers. In addition DDFSD pattern reductions $\left(\boldsymbol{\mu}=\left[\begin{array}{lll}1 & 1 & 1\end{array}\right]\right.$ and $\left.\boldsymbol{\mu}=\left[\begin{array}{ll}2 & 2\end{array}\right]\right)$ have the best performance for DRT but not for SRT; in fact we expect a DRT particularly effective for DDFSD since, as explained in sec. IV, double filtering is used to minimize the signal energy of the discarded taps.

Fig. 5 and 6 show similar performance results for the static channel decoded by 64 and 16 state trellises respectively: performance advantage provided by DRT is again more evident at largest state reductions. Differently from the previous channel, the best performance is provided by the pattern reductions $\boldsymbol{\mu}=\left[\begin{array}{lll}0 & 0 & 6\end{array}\right]$ for DRT or SRT and $\boldsymbol{\mu}=\left[\begin{array}{lll}0 & 0 & 4\end{array}\right]$ for DRT. It can be noted that flexible implementation of these complexity reduction techniques in

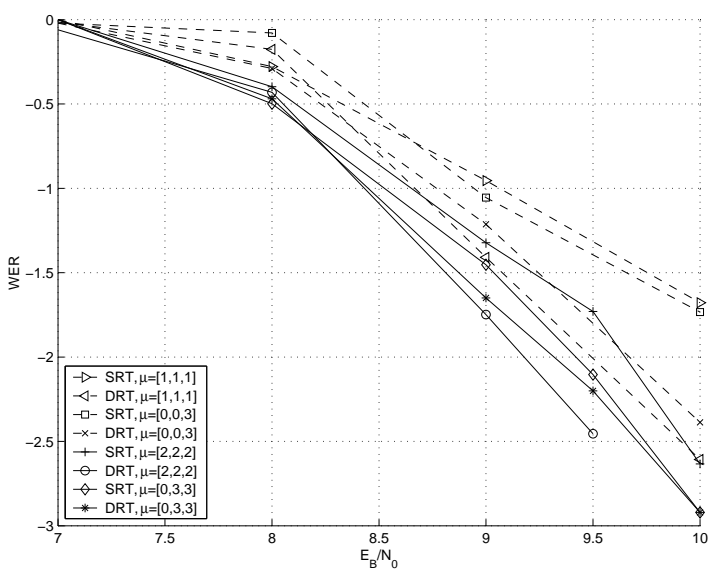

Fig. 4. Word error rate (WER) versus $E_{b} / N_{0}$ at the third iteration for the TU-GSM channel with channel SISO's reduced to 64 (continuous lines) and 8 states (dashed lines). 
8-PSK is fundamental to obtain a number of states that can be any power of 2 and not of 8 .

Of course performance improvement depends on the number of iterations, even if a small number equal to 3,4 is usually sufficient for achieving the final performance in these applications; DRT has usually a faster convergence and its use in a TEQ may be useful for limiting the decoding delay.

\section{Conclusions}

The paper has presented an architecture for implementing reduced state SISO equalizers in non binary modulations, maintaining full flexibility of the reduction patterns and the performance advantage provided by double pre-filtering techniques for forward and back-

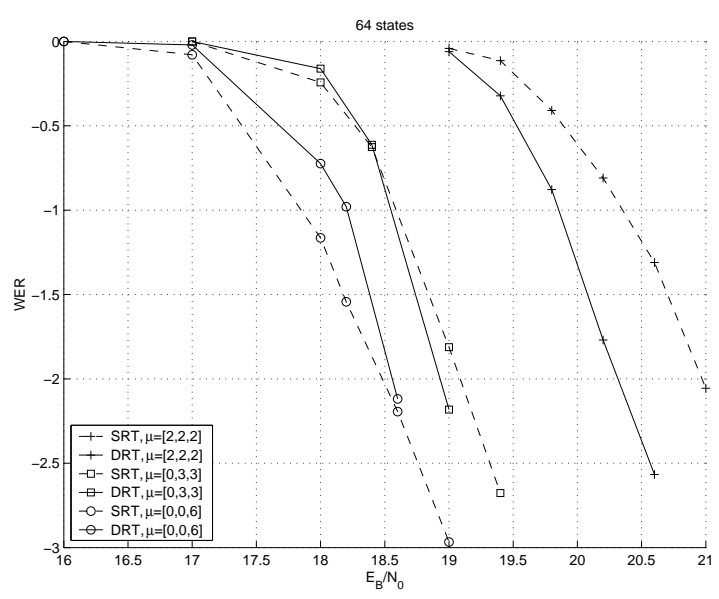

Fig. 5. Word error rate (WER) versus $E_{b} / N_{0}$ at the third iteration for the static channel with reduction to 64 states (SRT - dashed lines, DRT - continuous lines).

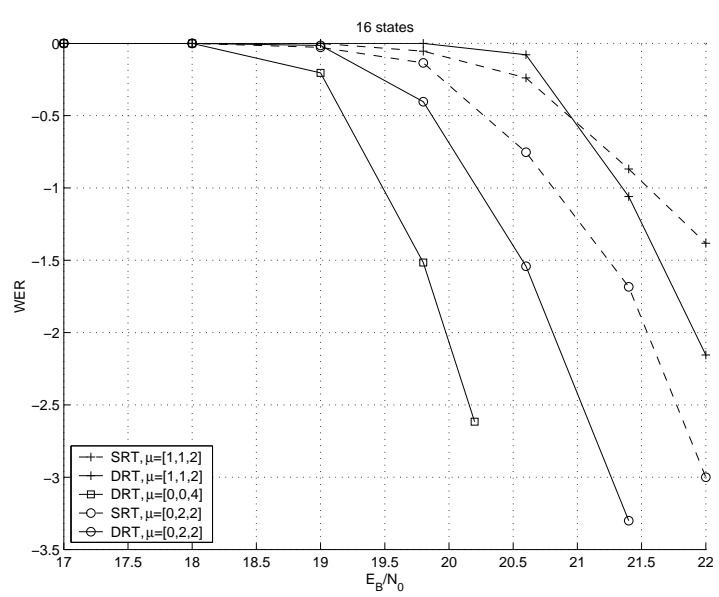

Fig. 6. Word error rate (WER) versus $E_{b} / N_{0}$ at the third iteration for the static channel with reduction to 16 states (SRT - dashed lines, DRT - continuous lines). The SRT scheme with $\boldsymbol{\mu}=\left[\begin{array}{lll}0 & 0 & 4\end{array}\right]$ does not provide satisfactory performance. ward recursions. Simulation results were presented for two RS-SISO equalizers and their corresponding performance were compared both for static and fading channels in a turbo equalizer scheme.

\section{REFERENCES}

[1] C. Douillard, M. Jezequel, C. Berrou, A. Picart, P. Didier, A. Glavieux, "Iterative correction of intersymbol interference: turbo equalization," European Trans. Telecommun., vol. 6, pp. 507-511, Sept.-Oct. 1995.

[2] K. Chugg, A. Anastasopoulos, X. Chen, "Iterative Detection," Kluwer Academic Publishers, 2001.

[3] M. V. Eyuboglu, S. U. H. Qureshi, "Reduced-state sequence estimation with set partitioning and decision feedback," IEEE Trans. Commun., vol. 36, pp. 13-20, Jan. 1988.

[4] G. Colavolpe, G. Ferrari, R. Raheli, "Reduced-state BCJR-type algorithms," IEEE J. Select. Areas Commun., vol. 19, pp. 848-859, May 2001.

[5] M. Magarini, L. Reggiani, A. Spalvieri, G. Tartara, "The benefits of the MMSE-DFE feedforward filter in reduced-complexity turbo equalization," Proc. ICT, 2003, pp. 1189-1193.

[6] D. Raphaeli, T. Kaitz, "A reduced-complexity algorithm for combined equalization and decoding," IEEE Trans. Commun., vol. 48, pp. 1797-1807, Nov. 2000.

[7] G. Ungerboeck, "Channel coding with multilevel/phase signals," IEEE Trans. Inform. Theory, vol. 28, pp. 55-67, Jan. 1982.

[8] W. H. Gerstacker, R. Schober, "Equalization concepts for EDGE," IEEE Trans. Wireless Commun., vol. 1, pp. 190-199, Jan. 2002.

[9] L. Szczecinski, I. Soto, "Is turbo equalization useful in EDGE systems?," Proc. VTC-Spring, 2002, pp. 80-84.

[10] "GSM Recommendations 05.05 Version 5.3.0," ETSI, Dec. 1996. 